\title{
UNDERWATER OPTICAL IMAGING: THE DESIGN OF OPTIMAL Systems
}

\author{
By Jules S. Jaffe
}

$\mathrm{U}$

NDERWATER optical imaging typically is limited by light scattering in the common volume of water intersected by the field of view of the camera and the illumination source. In such cases, the imaging system is contrast-limited. In other situations, the image contrast may be extremely high, however the amount of power falling upon the receiving sensor may be too small to be detectable; in this case the imaging system is power-limited.

The possibilities for long range underwater imaging are quite limited. Over a round-trip path, the intensity of a beam of light is exponentially attenuated by $\mathrm{e}^{-2 \mathrm{cr}}$, where $c$ is the total attenuation coefficient and $r$ is the distance from source to object. Assuming a kilowatt of photons at 488 nanometers, the number of attenuation lengths (e-folding distances) that would result in a single received photon is approximately 50 . Typical attenuation coefficients for deep ocean water, coastal water, and bay water are $0.05 \mathrm{~m}^{-1}, 0.2 \mathrm{~m}^{-1}$ and $0.33 \mathrm{~m}^{-1}$, respectively; these lead to depths of only $500 \mathrm{~m}, 125 \mathrm{~m}$ and $75 \mathrm{~m}$ over which one returning photon would be received. These distances are clearly overestimates of visibility, because they do not take into account the finite aperture of a camera, the reflectively of an underwater object, and the spreading of a light beam on its way toward an underwater object.

The basic compromise in underwater imaging design is between camera-to-light separation, contrast, and power, which all depend on environmental conditions. A convenient measure of the performance of an underwater imaging system is the range in total attenuation lengths at which the system provides acceptable images. Conventional systems that use coincident positioning of camera and lights can usually image well at 1 attenuation length, but they are contrast-limited at greater ranges. Systems with separated camera and lights usually can image at distances of up to 2-3 attenuation lengths. but at greater distances they succumb to backscatter. Typical separation distances on remotely-operated platforms are 3-5m (Harris and Ballard, 1986). If a distance greater than 3 attenuation lengths is desired.

Jules S. Jaffe, Marine Physical Laboratory A-f)(15. Soripps Insti tution of Oceanography, La Jolla, CA 92093. more exotic systems are necessary: examples are laser range-gated systems, and scanning light-beam systems. Fig. 1 schematically compares some traditional and exotic lighting systems.

\section{Computer Image Modeling}

Modeling of underwater imaging has been carried out with Monte Carlo techniques (Funk et. al.. 1972) and with a semianalytic Monte Carlo method adapted from the physics of scattering (Chilton et. al.. 1969). Gordon and Knittel (1973) formulated analytic equations that take into account multiple scattering. Their results agree well with the Monte Carlo simulations to a range of 6 attenuation lengths. The model discussed here, which is closely related to one formulated by (McGlamery, 1979), is a hybrid approach in which linearization is incorporated in certain situations in order to simplify the computation. When this approximation cannot be made, the predicted radiance fields are calculated explicitly.

An underwater imaging experiment consists of tracing the progression of light from a light source to a camera. The light received by the camera may include light that has been reflected by an object, and it may include light received without reflection from the object: the latter is called backscatter. Light reflected by the object that reaches the camera has two components: light that has not been scattered in the intervening water, and light that has been scattered at a small angle, called the forward-scattered component. A camera image therefore results from a linear superposition of backscattered, forward-scattered, and directly-reflected light.

Spherical spreading and attenuation of the source light beam is assumed in order to model the illumination incident upon the target plane. The reflected illumination is then computed as the product of the incident illumination and the reflectance map. Assuming a Lambertian reflector, geometric optics is then used to compute the image of the direct component in the camera plane. The reflected light is also small-angle scattered on its way to the camera. A fraction of the resultant blurred image is then added to the direct component.

The backscatter component is the most computationally demanding to calculate. The model partitions 3-dimensional space into planes parallel to the 


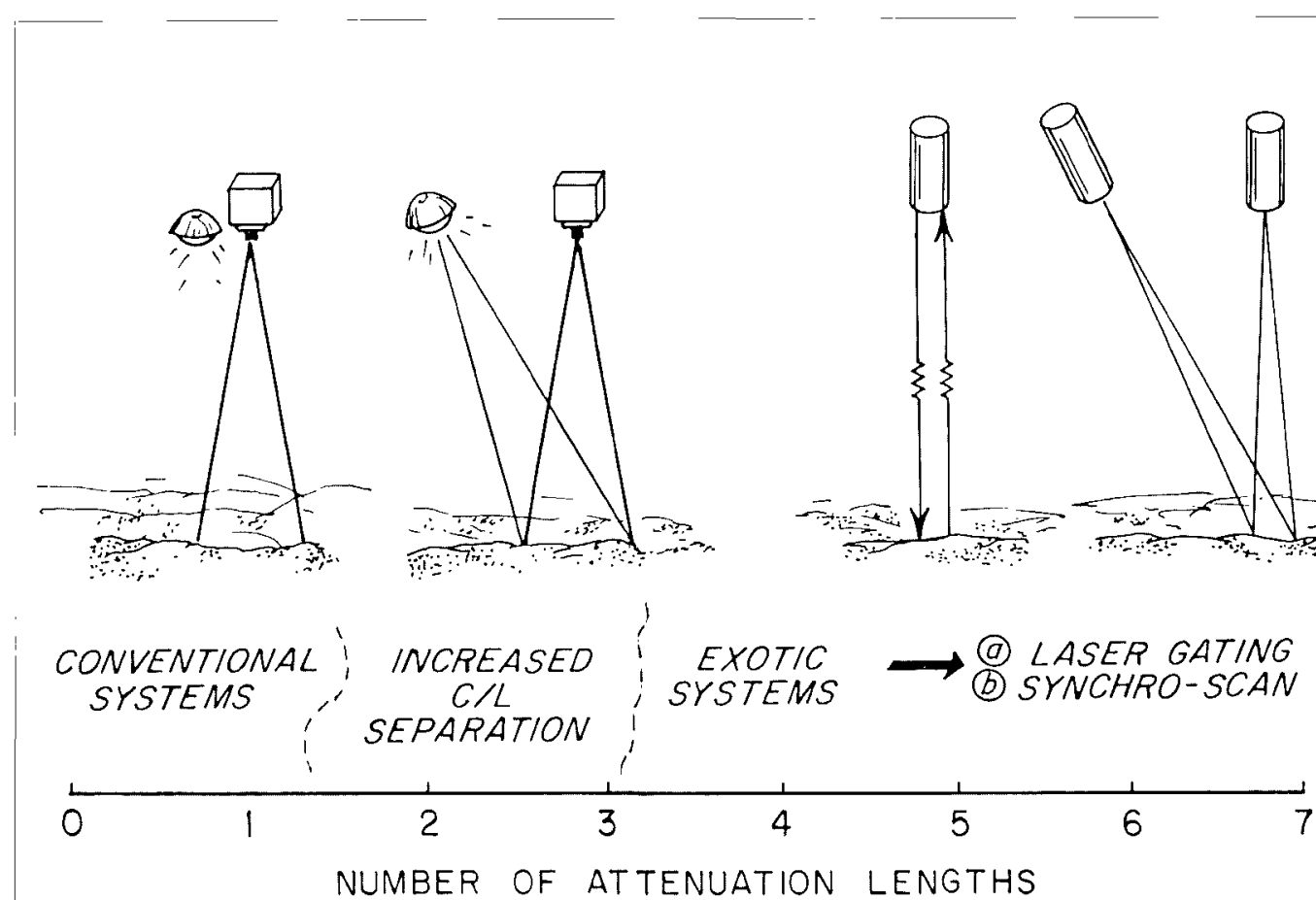

CONTRAST LIMITED
POWER LIMITED
Fig. 1

camera plane, and the radiation scattered toward the camera is computed superposing small volume elements weighted by an appropriate volume scattering function. Theoretical calculations indicate that the current model has validity to 6 attenuation lengths (Gordon and Knittel. 1973).

\section{Advanced Imaging Systems}

The computer model has been used to simulate an imaging system using a range-gated light source and receiver, as suggested by Heckman and Hodgson (1967). The method consists of propagating a pulse of light of short duration into the water, so that a slab of water of finite thickness is illuminated. If the receiving device is timed to be open for a time duration equal to that of the light pulse. then varying the onset time of the device limits the received information to that returned from the illuminated slab. If the distance to an object within the field of view is known, the receiver can be activated so that almost all of the reflected illumination and none of the backscatter will enter the camera.

The simulation suggests that range-gated imaging provides excellent contrast but is power-limited. With typical power levels, a laser range-gated system is limited to a performance of about 1 attenuation length in clear water $\left(\mathrm{c}^{-1}=20 \mathrm{~m}\right)$ and only 2 attenuation lengths in more "cloudy" water.

It is well known that better optical images can be produced by increasing the distance between the light source and the receiver, because the common backscatter volume between the two is reduced. Further improvement may be obtained by scanning an extremely narrow source beam along the axis that joins the camera and light source, digitally recording a sequence of images, and using image processing techniques to extract the high contrast components. It is then possible to synthesize a composite image that consists of a superposition of the individual images. An additional advantage of the method is that micro-bathymetry can be observed using computer vision techniques (Ballard and Brown, 1982).

Model simulations have shown that, with a vehicle of $3 \mathrm{~m}$ length and realistic lighting sources, objects may be observable in "cloudy" water conditions at distances of up to 5 attenuation lengths. Ongoing studies will define more thoroughly the actual limitations of the this technique in terms of power, contrast and image processing. Farly results suggest that the method has the potential to extend the range of underwater optical imaging systems to distances that were previously unobtainable.

\section{References}

Ballard, D.H. and D.M. Brown. 1982: Computer liston Prentice-Hall Inc. Englewood Cliffs, New Jersey, 523 pp.

Chilton. F.. D.D. Jones and W.K. Talley, 1969: Imaging Properties by the Sea, J. Opt. Soc. Am. 59. (8), $891-898$.

Funk. C.J., S.B. Bryant and P.J. Heckman Jr., 1972: Handbook of Underwater Imaging System Design, Naval Undersea Center, San Diego, CA, Rep. No. TP303.

Gordon. A. and M.R. Knittel, 1973: Underwater Multiple Scattering of Light for System Designers, Naval Undersea Center. San Diego. CA, Rep. No. TB371.

Harris, S.E. and R.D. Ballard, 1986: ARGO, Capabilities and Deep Ocean Explorations. Oceans ' $86^{\prime}, 1.6-8$.

Heckman, P. and R.T. Hodgson,1967: Underwater Optical Range Gating, IEEE J. of Quantum Elec.. QE-3, 11, 445-448.

McGlamery, B.J.. 1979: A Computer Model for Underwater Camera Systems. Ocean Optics VI, Proc. SPIE. 208 . (S.Q. Duntley, ed.), 221-231.
The receiver can be activated so that almost all of the reflected illumination and none of the backscatter will enter the camera. 